International Game Theory Review, Vol. 4, No. 2 (2002) 1-10

(C) World Scientific Publishing Company

\title{
NASH EQUILIBRIA IN LOAD BALANCING IN DISTRIBUTED COMPUTER SYSTEMS
}

\author{
EITAN ALTMAN \\ INRIA B.P. 93, 06902 Sophia Antipolis Cedex, France, and CESIMO, \\ Facultad de Ingenieria, Universidad de Los Andes, Mérida, Venezuela \\ eitan.altman@sophia.inria.fr \\ HISAO KAMEDA* and YOSHIHISA HOSOKAWA ${ }^{\dagger}$ \\ * The Institute of Information Sciences and Electronics, \\ ${ }^{\dagger}$ Graduate School of Systems and Information Engineering, \\ University of Tsukuba, Tsukuba Science City, Ibaraki 305-8573, Japan \\ *kameda@is.tsukuba.ac.jp \\ ${ }^{\dagger}$ hosokawa@osdp.is.tsukuba.ac.jp
}

\begin{abstract}
The use of game theoretical techniques has been quite successful in describing routing in networks, both in road traffic applications as well as in telecommunication networks applications. We study in this paper a third area of applications of such games, which is load balancing in distributed computer systems. One of the most important questions that arise in all applications of routing games is the existence and uniqueness of equilibrium. Whereas the existence of Nash equilibrium is known for general models of networks under weak assumptions, uniqueness results are only known for very special applications, i.e., either for very special cost functions or for very special topologies. We establish in this paper the uniqueness of an equilibrium for routing games with topologies that model well distributed computer systems, under quite general assumptions on the costs.
\end{abstract}

Keywords: Nash equilibrium; distributed computing; uniqueness.

\section{Introduction}

Game theoretic tools have been quite successful in the study of problems in which several users have to ship some objects in a network between sources and destinations. Two central application areas of such models are road traffic [see e.g., Dafermos (1972), Dafermos and Sparrow (1969), Haurie and Marcott (1985), Patriksson (1994), Wardrop (1952) and references therein] and telecommunication networks [see e.g., Altman et al. (2002), Altman, Başar and Srikant (1999), La and Anantharam (1997), Korilis, Lazar and Orda (1997), Orda, Rom and Shimkin (1993) and references therein]. A third quite new emerging area of application of routing games is distributed computing [Kameda et al. (2000), Kameda, Altman and Pourtallier (2000), Kameda and Pourtallier, Kameda et al. (1996), Kameda, Kozawa and Li (1997), Kameda and Zhang (1995), Kim and Kameda (1990), Li and 
Kameda (1998), Zhang, Kameda and Shimizu (1992)]. There are several different ways to model the decision making in non-cooperative routing. In the case that the number of decision makers is very large, and the influence of each decision maker on the performance of others is negligible, the optimality concept that has been used is known as the Wardrop equilibrium [Wardrop (1952)] (it is also called individual optimization, and some people call it user optimization). In contrast, if the number of decision makers is finite (and decisions of one player have nonnegligible influence on the performance of other players), the optimality concept that has been used is that of Nash-equilibrium (it is also called class optimization, and some people call it user optimization).

A fundamental question in the study of optimality solution concepts is whether they exist, and if they do, whether they are unique. In the setting of Wardrop equilibrium, it is well-known under fairly general conditions and for general topologies (suitable for the study of all the applications mentioned above), the equilibrium exists and is unique in the sense of global link flows [see e.g., Patriksson (1994) and references therein, as well as Altman and Kameda (2001) and Kameda and Zhang (1995)]. In the setting of Nash equilibrium too, the existence of a Nash equilibrium is known under general conditions. However, the equilibrium may not be unique (even in the sense of global link flows), as was shown in a simple counterexample in Orda, Rom and Shimkin (1993). Uniqueness of the Nash equilibrium has been established for either special cost functions [Altman et al. (2002)], or for very restrictive topologies [Orda, Rom and Shimkin (1993)] (the special case of parallel links), or under other particular assumptions [Altman and Kameda (2001) and Orda, Rom and Shimkin (1993).] [We note that the classical approach of Rosen (1965) is only rarely useful in routing games; indeed, even for the simple case of a network that consists of only two parallel links, it only works for very light traffic, see Orda, Rom and Shimkin (1993).] Due to these restrictions, we do not have a general theory that can apply to different application areas. In this paper we shall investigate routing games in several topologies that model distributed computing. We shall establish uniqueness of the Nash equilibrium under general assumptions on the costs.

\section{The Model and Assumptions}

We consider a model consisting of $K$ nodes (hosts) and a communication means that connects the nodes. Nodes are numbered $i=1, \ldots, K$. Each node consists of a single server with service rate $\mu_{i}(i=1, \ldots, K)$. Let there be $K$ sets of classes: $\mathcal{K}_{i}(i=1, \ldots, K)$ where $\mathcal{K}_{i}$ contains $K_{i}(i=1, \ldots, K)$ classes of users. The set $\mathcal{K}_{i}$ corresponds to the users that send flow originally to node $i$. Each of these users can decide to route part of his flow to node $j, j \neq i$ through a communication line. We consider the problem of uniqueness of the equilibrium in which each one of the classes minimizes its own average delay. Denote $\mathcal{K}=\bigcup_{i=1}^{K} \mathcal{K}_{i}$. 
We introduce the following notation:

$\phi^{(i, r)}$ the input arrival rate of class $(i, r)$.

$x_{i i}^{(i, r)}$ the flow originating at node $i$ by class $(i, r)$ that is processed at node $i$.

$x_{i j}^{(i, r)}$ the flow originating at node $i$ by class $(i, r)$ that is processed at node $j$.

$\mathbf{x} \quad$ the set of all flows: $\mathbf{x}=\left(x_{i j}^{(i, r)}, i=1, \ldots, K, j=1, \ldots, K,(i, r) \in \mathcal{K}\right)$.

$\beta_{i} \quad$ the total load on node $i$, i.e., $\beta_{i}=\sum_{j=1}^{K} \sum_{(j, r) \in \mathcal{K}_{j}} x_{j i}^{(j, r)}$.

$\lambda_{i j} \quad$ the total flow forwarded from node $i$ to node $j: \lambda_{i j}=\sum_{(i, r) \in \mathcal{K}_{i}} x_{i j}^{(i, r)}$.

$\lambda \quad$ the total network flow, i.e., $\lambda=\sum_{i=1}^{K} \sum_{j \neq i} \lambda_{i j}$.

We have $\sum_{j=1}^{K} x_{i j}^{(i, r)}=\phi^{(i, r)}, x_{i j}^{(i, r)} \geq 0$. We denote the $x$ 's that satisfy the constraints by $C$. In Kameda et al. (2000), for example, the expected processing (including queueing) time of a job that is processed at node $i$, was taken to be $1 /\left(\mu_{i}-\beta_{i}\right)$ for $\beta_{i}<\mu_{i}$ (otherwise it is infinite).

As to the communication means, we consider two alternatives.

(A) The one is a single-channel communication line that is used commonly in forwarding and sending back of jobs that arrive at both nodes.

(B) The other consists of two-way communication lines between each two links. One two-way line $i j$ is used for forwarding of a job that arrives at node $i$ and is forwarded to node $j$ (and for sending back the processed result of the job).

We define $L$ to be the set of system elements; in case (A), $L$ contains the set of nodes as well as the communication bus $L_{A}=\{1, \ldots, K, c\}$ (where $c$ stands for the communication bus). In case (B), $L$ contains the set of nodes as well as the set of unidirectional links $L_{B}=\{1, \ldots, K,(i j), i, j=1, \ldots, K, j \neq i\}$.

Associated with each system element $l \in L$ and each class $(i, r)$, there is a cost $J_{l}^{(i, r)}(\mathbf{x})$.

Let $J^{(i, r)}(\mathbf{x})$ denote the cost of class $(i, r)$. A typical cost often considered in the literature is the mean response time, or mean delay. More precisely, we refer to the length of time between the instant when a job arrives at a node and the
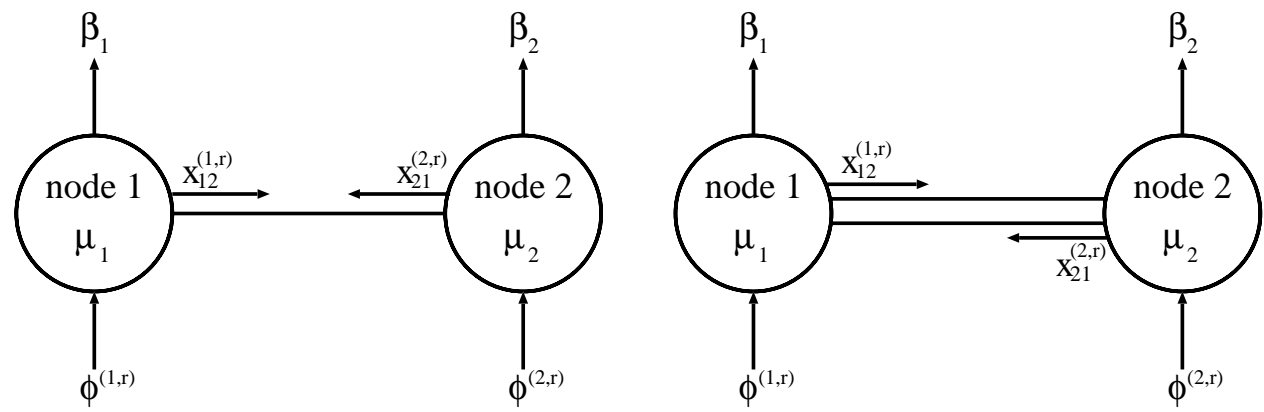

Fig. 1. The system model, case (A) (left) and case (B) (right) for two nodes and two classes. 
instant when a job leaves the node, where it has arrived, after all processing and communication, if any, are over as the response time for a job arriving at the node. The mean response time for class $(i, r)$ is then given by averaging the response time over all the jobs of that class. We make the following assumptions on the cost, which ensure the existence of an equilibrium.

G1 $J^{(i, r)}$ is the sum of the local processing cost and the communication cost for class $(i, r)$ where the latter are only functions of their local flow rate, i.e., in case (A) we have

$$
J^{(i, r)}(\mathbf{x})=\sum_{i=1}^{K} J_{i}^{(i, r)}(\mathbf{x})+J_{c}^{(i, r)}(\mathbf{x})
$$

and for case (B) we have for $(i, r) \in \mathcal{K}_{i}$

$$
J^{(i, r)}(\mathbf{x})=\sum_{j=1}^{K} J_{j}^{(i, r)}(\mathbf{x})+\sum_{j \neq i} J_{i j}^{(i, r)}\left(x_{i j}^{(i, r)}\right) .
$$

G2 $J_{l}^{(i, r)}$ are continuous functions whose range is the nonnegative quadrant and their image is $[0, \infty]$.

G3 $J_{l}^{(i, r)}$ are convex functions in the rate sent by class $(i, r)$ over the system element $l$. For example, if $l$ is the processor $i$ and $(i, r) \in \mathcal{K}_{i}$ then $J_{i}^{(i, r)}$ is assumed to be convex in $x_{i i}^{(i, r)}$.

G4 Whenever finite, $J_{l}^{(i, r)}$ is continuously differentiable in the flow sent by user $(i, r)$ to system element $l$. We denote $K_{l}^{(i, r)}(\mathbf{x})$ the partial derivative of $J_{l}^{(i, r)}(\mathbf{x})$ with respect to the flow sent by user $(i, r)$ to system element $l$.

G5 If not all classes have finite cost and one of the classes has infinite cost then it can change its own flow to make this cost finite.

G5 ensures that any equilibrium has finite costs for all players.

Lemma 2.1. Under conditions G1-G5 there exists an equilibrium.

Proof. The proof follows from Theorem 1 in Rosen (1965) [see also Orda, Rom and Shimkin (1993)].

We introduce the following further assumptions on the cost that will be used to establish uniqueness.

$\left(\Pi_{1}\right) K_{l}^{(i, r)}$ is a function of two arguments: (i) the total flow on the system element $l$, and (ii) the flow that class $(i, r)$ sends to node element $l . K_{l}^{(i, r)}$ is strictly increasing in each of its two arguments. 


\section{Uniqueness for Case (A)}

For a fixed assignment of the other class, class $(i, r)$ is faced with a constrained minimization problem. Its associated Lagrangian is given by

$$
\Lambda^{(i, r)}(\mathbf{x})=J^{(i, r)}(\mathbf{x})-\alpha^{(i, r)}\left(\sum_{j=1}^{K} x_{i j}^{(i, r)}-\phi^{(i, r)}\right) .
$$

$\mathbf{x}^{*}$ is thus an equilibrium if and only if it satisfies the Kuhn-Tucker conditions [see Shapiro (1979), pp. 158-165]: There exist some real numbers $\alpha^{(i, r)},(i, r) \in \mathcal{K}$ such that for $(i, r) \in \mathcal{K}_{i}, i=1,2$ :

$$
\begin{gathered}
K_{i}^{(i, r)}\left(\mathbf{x}^{*}\right) \geq \alpha^{(i, r)} \text { and } K_{i}^{(i, r)}\left(\mathbf{x}^{*}\right)=\alpha^{(i, r)} \text { if } x_{i i}^{(i, r)}>0 \\
K_{c}^{(i, r)}\left(\mathbf{x}^{*}\right)+K_{j}^{(i, r)}\left(\mathbf{x}^{*}\right) \geq \alpha^{(i, r)} \text { and } K_{c}^{(i, r)}\left(\mathbf{x}^{*}\right)+K_{j}^{(i, r)}\left(\mathbf{x}^{*}\right)=\alpha^{(i, r)} \text { if } x_{i j}^{(i, r)}>0 \\
x_{i i}^{(i, r)}, x_{i j}^{(i, r)} \geq 0, \quad \sum_{j=1}^{K} x_{i j}^{(i, r)}=\phi^{(i, r)} .
\end{gathered}
$$

We shall consider in this section only the case of two nodes and a single bidirectional communicating link between them. (We allow for several classes to arrive at each one of the two nodes.)

Theorem 3.1. The node optimization has a unique solution under assumption $\left(\Pi_{1}\right)$.

Proof. Let $\hat{\mathbf{x}}$ and $\mathbf{x}$ be two equilibria such that

$$
\hat{\lambda} \geq \lambda \text {. }
$$

Let $i$ be such that $\hat{\beta}_{i} \geq \beta_{i}$. We shall show that for all $(j, r) \in \mathcal{K}_{j}$,

$$
\hat{x}_{j j}^{(j, r)} \geq x_{j j}^{(j, r)}, \quad \text { or equivalently, } \hat{x}_{j i}^{(j, r)} \leq x_{j i}^{(j, r)}
$$

(the equivalence follows from the constraint Eq. (1) on the sum of the flows.) Equation (3) holds trivially if $x_{j j}^{(j, r)}=0$, so we have to check only the case $x_{j j}^{(j, r)}>0$. To do so, fix some $(j, r) \in \mathcal{K}_{j}$ and consider the following two subcases. Assume that

(a) $\hat{\alpha}^{(j, r)} \geq \alpha^{(j, r)}$. Note that $\hat{\beta}_{i} \geq \beta_{i}$ is equivalent to $\hat{\beta}_{j} \leq \beta_{j}$ (the equivalence follows from the constraint Eq. (1)). Hence

$$
K_{j}^{(j, r)}\left(\hat{x}_{j j}^{(j, r)}, \hat{\beta}_{j}\right) \geq \hat{\alpha}^{(j, r)} \geq \alpha^{(j, r)}=K_{j}^{(j, r)}\left(x_{j j}^{(j, r)}, \beta_{j}\right) \geq K_{j}^{(j, r)}\left(x_{j j}^{(j, r)}, \hat{\beta}_{j}\right) .
$$

The equality as well as the second inequality follow from the Kuhn-Tucker conditions, whereas the last inequality follows from the monotonicity assumption $\left(\Pi_{1}\right)$. Using again the monotonicity assumption $\left(\Pi_{1}\right)$, this time for the first argument, we conclude from the fact $K_{j}^{(j, r)}\left(\hat{x}_{j j}^{(j, r)}, \hat{\beta}_{j}\right) \geq K_{j}^{(j, r)}\left(x_{j j}^{(j, r)}, \hat{\beta}_{j}\right)$ that Eq. (3) holds. 
Thus we try instead of (a):

(b) $\hat{\alpha}^{(j, r)} \leq \alpha^{(j, r)}$. Equation (3) holds trivially if $\hat{x}_{j i}^{(j, r)}=0$, since in this case $\hat{x}_{j j}^{(j, r)}=\phi^{(j, r)} \geq x_{j j}^{(j, r)}$. So it remains to check the case $\hat{x}_{j i}^{(j, r)}>0$. We then have for $(j, r) \in \mathcal{K}_{j}$

$$
\begin{aligned}
& K_{c}^{(j, r)}\left(x_{j i}^{(j, r)}, \lambda\right)+K_{i}^{(j, r)}\left(x_{j i}^{(j, r)}, \beta_{i}\right) \\
& \quad \geq \alpha^{(j, r)} \geq \hat{\alpha}^{(j, r)}=K_{c}^{(j, r)}\left(\hat{x}_{j i}^{(j, r)}, \hat{\lambda}\right)+K_{i}^{(j, r)}\left(\hat{x}_{j i}^{(j, r)}, \hat{\beta}_{i}\right) \\
& \quad \geq K_{c}^{(j, r)}\left(\hat{x}_{j i}^{(j, r)}, \lambda\right)+K_{i}^{(j, r)}\left(\hat{x}_{j i}^{(j, r)}, \beta_{i}\right) .
\end{aligned}
$$

Here, the first inequality and the equality follow from the Kuhn-Tucker conditions, whereas the last inequality follows from the monotonicity of $K_{l}^{(j, r)}$ (property $\left(\Pi_{1}\right)$ ). Using again the monotonicity, we conclude that Eq. (3) holds in case (B) as well.

We conclude that

$$
\sum_{(j, r) \in \mathcal{K}_{j}} \hat{x}_{j i}^{(j, r)} \leq \sum_{(j, r) \in \mathcal{K}_{j}} x_{j i}^{(j, r)} .
$$

Combining this with $\hat{\beta}_{i} \geq \beta_{i}$, we conclude that

$$
\sum_{(i, r) \in \mathcal{K}_{i}} \hat{x}_{i i}^{(i, r)} \geq \sum_{(i, r) \in \mathcal{K}_{i}} x_{i i}^{(i, r)}
$$

But since for $(i, r) \in \mathcal{K}_{i}, \hat{x}_{i i}^{(i, r)}+\hat{x}_{i j}^{(i, r)}=\phi^{(i, r)}$, it follows that

$$
\sum_{(i, r) \in \mathcal{K}_{i}} \hat{x}_{i j}^{(i, r)} \leq \sum_{(i, r) \in \mathcal{K}_{i}} x_{i j}^{(i, r)}
$$

Combining this with Eq. (5) we conclude that $\hat{\lambda} \leq \lambda$. This contradicts our assumption Eq. (2), unless we have equality in Eq. (2).

This implies in particular that Eq. (3) holds (as we derived above). Now, if for some $(j, r) \in \mathcal{K}_{j}$ Eq. (3) holds with strict inequality then Eqs. (5) and (6) would hold with strict inequality, which would imply that $\hat{\lambda}<\lambda$. But since we established that Eq. (2) holds with equality, we conclude that Eq. (3) holds with equality. By a symmetric argument (the one in the paragraph below Eq. (6)) we establish Eq. (3) also for $(i, r) \in \mathcal{K}_{i}$. We conclude that $\mathbf{x}=\hat{x}$.

An interesting property that can be obtained from the above proof is that not only the Nash equilibrium is unique, but also:

Lemma 3.1. There are unique Lagrange multipliers for the node optimization under assumption $\left(\Pi_{1}\right)$.

Proof. Assume that there are two sets of Lagrange multipliers, $\alpha$ and $\hat{\alpha}$ corresponding to the Nash equilibria $\mathbf{x}$ and $\hat{\mathbf{x}}$ (where $\mathbf{x}=\hat{\mathbf{x}}$ due to the uniqueness). Assume that there are some $r, j$ for which $\hat{\alpha}^{(j, r)}>\alpha^{(j, r)},(j, r) \in \mathcal{K}_{j}$. It follows 
that Eq. (4) holds with the first inequality being a strict one. Since $K_{j}^{(j, r)}$ is strictly monotone in both arguments, it follows from Eq. (4) that $\hat{x}_{j j}^{(j, r)}>x_{j j}^{(j, r)}$. This contradicts, however, the uniqueness of the Nash equilibrium.

\section{Uniqueness of the Node Optimum for Case (B)}

We allow in this section for arbitrary numbers of nodes and classes.

We impose the following restriction on the model:

$\left(\Pi_{2}\right)$ A class $(i, r) \in \mathcal{K}_{i}$ that decides to ship some flow to a node $j \neq i$ should do it using a single hop (a single communication link); it is not possible for it to use two hops ( $i$ to $k$ and then $k$ to $j$ ), if there is a link that connect nodes $i$ and $j$ directly.

Remark 4.1. Assumption $\left(\Pi_{2}\right)$ is frequently used in load balancing in distributed computer systems. See Kameda et al. (1996).

The proof is done by transforming our problem into the following equivalent routing problem for which the uniqueness is known.

Consider a network $\mathcal{G}$ consisting of two nodes: $a$ and $b$, and of $K$ parallel directed links, all from node $a$ to node $b$. There are $K$ classes of flows $\mathcal{K}_{i}, i=1, \ldots, K$, all having node $a$ as the source and node $b$ as the destination. The total rate of class $(i, r)$ is $\phi^{(i, r)}$.

Let $\mathrm{x}_{l}^{(i, r)}$ be the rate at which class $(i, r)$ sends over link $l(l=1,2, \ldots, K)$. These quantities satisfy the constraints $x_{l}^{(i, r)} \geq 0, l=1, \ldots, K$ and $\sum_{i} x_{i}^{(i, r)}=\phi^{(i, r)}$. Class $(i, r)$ determines $\left(\mathrm{x}_{1}^{(i, r)}, \ldots, \mathrm{x}_{K}^{(i, r)}\right)$ so as to minimize its cost $\bar{J}^{(i, r)}(\overline{\mathbf{x}})$, where $\overline{\mathbf{x}}=\left(\mathrm{x}_{l}^{(i, r)}, l \in L,(i, r) \in \mathcal{K}\right)$. Define $\mathrm{x}_{l}=\sum_{(i, r) \in \mathcal{K}} \mathrm{x}_{l}^{(i, r)}$.

We assume that $\bar{J}^{(i, r)}$ is the sum of the link cost functions:

$$
\bar{J}^{(i, r)}(\overline{\mathbf{x}})=\sum_{l} \bar{J}_{l}^{(i, r)}(\overline{\mathbf{x}}),
$$

where $\bar{J}_{p}^{(i, r)}(\overline{\mathbf{x}})$ are expressed in terms of the costs $\left(J_{l}^{(i, r)}\right)$ defined in Sec. 2 as follows. For any $i$ and any $(i, r) \in \mathcal{K}_{i}$,

$$
\begin{aligned}
& \bar{J}_{i}^{(i, r)}(\overline{\mathbf{x}})=J_{i}^{(i, r)}\left(\mathrm{x}_{i}^{(i, r)}, \mathrm{x}_{i}\right), \\
& \bar{J}_{j}^{(i, r)}(\overline{\mathbf{x}})=J_{i j}^{(i, r)}\left(\mathrm{x}_{j}^{(i, r)}, \mathrm{x}_{j}\right)+J_{j}^{(i, r)}\left(\mathrm{x}_{j}^{(i, r)}, \mathrm{x}_{j}\right) \text { for } j \neq i .
\end{aligned}
$$

If the costs for the original load balancing problem satisfy assumption $\left(\Pi_{1}\right)$, it follows that the costs for the new routing problem also do. The routing problem has a unique Nash equilibrium under assumption $\left(\Pi_{1}\right)$. See Theorem 1 in Orda, Rom and Shimkin (1993) (that theorem states some other assumptions which are not used in its proof). By identifying the decision variables $\overline{\mathbf{x}}$ in the new routing problem with the decision variables $\mathbf{x}$ in the original load balancing problem, we see that the minimization problems faced by each class is the same in both cases, 
and therefore we conclude that the node optimum in our original problem exists and is unique.

\section{Some Properties of the Node Equilibrium}

We use the model, the notation as well as the assumptions $\mathrm{G}$ and $\left(\Pi_{1}\right)$ on the cost, described in Sec. 2. We further assume $\left(\Pi_{2}\right)$ (introduced in the previous section).

Assume further that the costs $J_{l}^{(i, r)}$ of the $l$ th system element are given by the product of the amount of flow that class $(i, r)$ ships through that element and the average delay $T_{l}$ in this element. For example, for $(i, r) \in \mathcal{K}_{i}$ we have

$$
J_{i}^{(i, r)}(\mathbf{x})=x_{i i}^{(i, r)} T_{i}\left(\beta_{i}\right) .
$$

We further assume that for each $l$, the partial derivative of $T_{l}$ with respect to the total flow through that element is strictly positive. In other words, for every node $i$,

$$
\partial T_{i}\left(\beta_{i}\right) / \partial \beta_{i}>0,
$$

with similar expressions for the communication costs.

Theorem 5.1. Consider either case (A) or case (B). Let $\phi^{(i, r)}>0$ for all classes.

(i) Assume that at equilibrium, all the traffic of some class $(i, r) \in \mathcal{K}_{i}$ arriving to node $i$ is routed away from that node.

Let $j$ be another node to which some positive flow is routed by class $(i, r)$. Consider now any $(j, s) \in \mathcal{K}_{j}$. If $(j, s)$ sends some positive amount of flow to node $i$ then it also processes some positive amount of flow at node $j$.

(ii) Consider the case of two nodes $K=2$, and assume that $\phi^{(1, r)} \geq \phi^{(2, s)},(1, r) \in$ $\mathcal{K}_{1},(2, s) \in \mathcal{K}_{2}$. Assume that $(2, s)$ sends at equilibrium some flow to node 1 .

Then at equilibrium, class $(1, r)$ sends some strictly positive flow to be processed in node 1.

Proof. We shall prove for case (B); the proof for case (A) is the same except that the $K_{i j}$ below should be replaced by $K_{c}$.

(i) Using the Kuhn-Tucker conditions for class 1 we have

$$
\begin{aligned}
K_{i}^{(i, r)}\left(x_{i i}^{(i, r)}, \beta_{i}\right) & =T_{i}\left(\beta_{i}\right) \geq \alpha^{(i, r)}=K_{i j}^{(i, r)}\left(x_{i j}^{(i, r)}, \lambda_{i j}\right)+K_{j}^{(i, r)}\left(x_{i j}^{(i, r)}, \beta_{j}\right) \\
& =K_{i j}^{(i, r)}\left(x_{i j}^{(i, r)}, \lambda_{i j}\right)+x_{i j}^{(i, r)} \partial T_{j}\left(\beta_{j}\right) / \partial \beta_{j}+T_{j}\left(\beta_{j}\right) .
\end{aligned}
$$

Combining this with assumption (7) we conclude that

$$
T_{i}\left(\beta_{i}\right)>T_{j}\left(\beta_{j}\right) .
$$

Assume that all traffic of source $(j, s)$ is routed away from some source $(j, s)$ that sends positive flow to node $i$. By using the Kuhn-Tucker conditions for class $(j, s)$ we get by the same arguments as above $T_{i}\left(\beta_{i}\right)<T_{j}\left(\beta_{j}\right)$, which gives a contradiction. This establishes the proof. 
(ii) Assume $\phi^{(1, r)} \geq \phi^{(2, s)}$, and assume that class $(1, r)$ sends all its traffic to node 2. We have by Eq. (8)

$$
\begin{aligned}
T_{1}\left(\beta_{1}\right) & \geq \alpha^{(1, r)} \geq K_{12}^{(1, r)}\left(x_{12}^{(1, r)}, \lambda_{12}\right)+\phi^{(1, r)} \partial T_{2}\left(\beta_{2}\right) / \partial \beta_{2}+T_{2}\left(\beta_{2}\right) \\
& >K_{12}^{(1, r)}\left(x_{12}^{(1, r)}, \lambda_{12}\right)+x_{22}^{(2, s)} \partial T_{2}\left(\beta_{2}\right) / \partial \beta_{2}+T_{2}\left(\beta_{2}\right) \\
& =K_{12}^{(1, r)}\left(x_{12}^{(1, r)}, \lambda_{12}\right)+K_{2}^{(2, s)}\left(x_{22}^{(2, s)}, \beta_{2}\right) \geq K_{2}^{(2, s)}\left(x_{22}^{(2, s)}, \beta_{2}\right) \\
& \geq \alpha^{(2, s)} \geq T_{1}\left(\beta_{1}\right) .
\end{aligned}
$$

The inequality Eq. (9) follows since $\phi^{(1, r)} \geq \phi^{(2, s)}>x_{22}^{(2, s)}$. The inequality before the last follows by the Kuhn-Tucker conditions. The last inequality is obtained similarly to Eq. (8) (and using the fact that class $(2, s)$ sends nonzero flow to node 1 ). We thus obtained a contradiction, which establishes the proof.

\section{Concluding Remarks}

In this paper, we showed the existence and uniqueness of Nash equilibria for routing games that arise in load balancing in distributed computing. We considered two scenarios that differ in the architecture of the communications means between the processors. Our results were obtained for quite general costs, thus extending the class of networks for which we know that the equilibrium is unique.

\section{References}

Altman, E., T. Başar, T. Jiménez and N. Shimkin (2002). "Competitive routing in networks with polynomial cost," IEEE Trans. Automatic Control, Vol. 47, 92-96.

Altman, E., T. Başar and R. Srikant (1999). "Nash equilibria for combined flow control and routing in networks: Asymptotic behavior for a large number of users," Proc. 38th IEEE Conference on Decision and Control (Phoenix, Arizona, USA, December, 1999).

Altman, E. and H. Kameda (2001). "Equilibria for multiclass routing in multi-agent networks," Proc. 40th IEEE Conference on Decision and Control (Orlando, Florida, December, 2001), 604-609.

Dafermos, S. (1972). "The traffic assignment problem for multiclass-user transportation networks," Transportation Sci., Vol. 6, 73-87.

Dafermos, S. and F. T. Sparrow (1999). "The traffic assignment problem for a general network," J. Research of the National Bureau of Standards-B. Math. Sci., 91-118.

Haurie, A. and P. Marcott (1985). "On the relationship between Nash-Cournot and Wardrop equilibria," Networks, Vol. 15, 295-308.

Kameda, H., E. Altman, T. Kozawa and Y. Hosokawa (2000). "Braess-like paradoxes in distributed computer systems," IEEE Trans. Automatic Control, Vol. 45, 1687-1691.

Kameda H., E. Altman and O. Pourtallier (2000). "Analytic study of mixed optima in symmetric distributed computer systems," Proc. 9th International Symposium on Dynamic Games and Applications (Adelaide, Australia, December 18-21, 2000).

Kameda, H. and O. Pourtallier. "Paradoxes in distributed decisions on optimal load balancing for networks of homogeneous computers," J. ACM (to appear). 
Kameda, H., J. Li, C. Kim and Y. Zhang (1996). Optimal Load Balancing in Distributed Computer Systems, Springer.

Kameda, H., T. Kozawa and J. Li (1997). "Anomalous relations among various performance objectives in distributed computer systems," Proc. 1st World Congress on Systems Simulation, IEEE, 459-465.

Kameda, H. and Y. Zhang (1995). "Uniqueness of the solution for optimal static routing in open BCMP queueing networks," Mathematical and Computer Modelling, Vol. 22, No. $10-12,119-130$.

Kim, C. and H. Kameda (1990). "An Algorithm for optimal static load balancing in distributed computer systems," IEEE Trans. Compt., Vol. 41, No. 3, 381-384.

Kim, C. and H. Kameda (1990). "Optimal static load balancing of multi-class jobs in a distributed computer system," Proc. 10th Intl. Conf. Distributed Comput. Syst., IEEE, 562-569.

La, R. J. and V. Anantharam (1997). "Optimal routing control: Game theoretic approach," Proc. 36th IEEE Conference on Decision and Control (San Diego, California).

$\mathrm{Li}$, J. and H. Kameda (1998). "Load balancing problems for multiclass jobs in distributed/parallel computer systems," IEEE Trans. Compt., Vol. 47, No. 3, 322-332.

Korilis, Y. A., A. A. Lazar and A. Orda (1997). "Capacity allocation under noncooperative routing," IEEE Trans. Automatic Control, Vol. 42, No. 3, 309-871.

Korilis, Y. A., A. A. Lazar and A. Orda (1997). "Avoiding the Braess paradox in noncooperative networks," Proc. IEEE Conference on Decision and Control (San Diego), 864-878.

Orda, A., R. Rom and N. Shimkin (1993). "Competitive routing in multiuser communication networks," IEEE/ACM Trans. Networking, Vol. 1, 614-627.

Patriksson, M. (1994). The Traffic Assignment Problem: Models and Methods (VSP BV, P.O. Box 346, 3700 AH Zeist, The Netherlands).

Rosen, J. B. (1965). "Existence and uniqueness of equilibrium points for concave N-person games," Econometrica, Vo.l 33, 153-163.

Shapiro, J. F. (1979). Mathematical Programming, Structures and Algorithms (J. Wiley and Sons).

Wardrop, J. G. (1952). "Some theoretical aspects of road traffic research," Proc. Inst. Civ. Eng. Part 2, 325-378.

Zhang, Y., H. Kameda and K. Shimizu (1992). "Parametric analysis of optimal load balancing in distributed computer systems," J. Information Processing 14, Vol. 4, 433-441. 